\title{
Demenzstationen stellen keine sinnvolle Spezialisierung dar - Kontra
}

\author{
Dementia Wards are not a Useful Specialisation - Contra
}

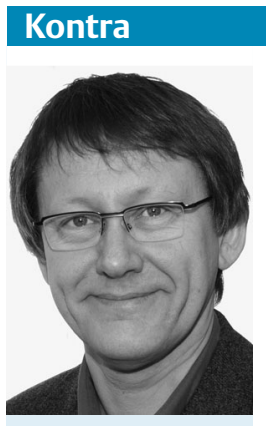

Jochen Tenter
Die störungsspezifi-

sche Behandlung in

der psychiatrischen

Arbeit kann ohne

Übertreibung als der

größte Entwicklungsschritt der letz-

ten 50 Jahre gelten, sofern man die Entwicklung ambulanter, aufsuchender oder teilstationärer

Einrichtungen sowie zahlreiche nicht psychiatrische kommunale Angebote miteinbezieht. Kognitive Verhaltenstherapie bei Panikstörung, Psychoedukation bzw. kognitive Verfahren bei Psychosen, suchttherapeutische Konzepte und viele andere mehr haben sich als effektiver als eine allgemein unterstützende und empathische Gesprächsführung herausgestellt.

Als sich in den frühen 90er-Jahren die Leiter gerontopsychiatrischer Abteilungen an den Fachkrankenhäusern trafen, stand die Verbesserung der Behandlung von Demenzpatienten und Betroffenen, die an den Herausforderungen des Älterwerdens scheitern („Altersdepression“) im Vordergrund. Aus diesem Kreis entwickelte sich die heutige Fachgesellschaft DGGPP. Bis dato waren Alterspsychiatrien eher Pflegestationen oder Sterbestationen, oft der Inneren Medizin zugeordnet. Inzwischen gibt es ein umfangreiches Wissen und Strategien, um multimorbide Ältere, unter denen Delir- und Demenzpatienten die größte Gruppe stellen, fachgerecht und spezifisch zu behandeln. Wissen und Weiterentwicklung gingen maßgeblich von den differenzierten gerontopsychiatrischen Abteilungen an Fachkrankenhäusern aus.

Bereits 1995 wurde auf dem Kongress der DGGPP in einem Symposium das Thema in einer Pro- („Demenzstationen“) und Kontra-Debatte bearbeitet [1]. Schon damals wurde vorausgesehen, dass nur noch wenige, dann aber sehr schwer Demenzkranke stationär behandelt werden müssen. Dafür wurden optimale spezifische Rahmenbedingen gefordert, z.B. architektonisch und bezüglich Schulung der Mitarbeitenden.

Die Gestaltung des Milieus ist von entscheidender Bedeutung. Die Pflege benötigt ein gemeinsames Konzept des Umgangs und der Kommunikation, das besonders wirksam ist, wenn alle gleich geschult sind und sich gegenseitig im Umgang bestärken. Dies hat ebenso die Altenhilfe erkannt und sich im Verlauf der letzten 20 Jahre professionalisiert und das Milieu für Demenzkranke, die zwei Drittel bis drei Viertel der Bewohner von Pflegeheimen stellen, optimiert. Dass sich inzwischen dort nicht an Demenz Erkrankte teils etwas verloren fühlen, unterstreicht, wie unterschiedlich die Milieus gestaltet werden müssen. Die Altenhilfe hat ihre Neubauten demenzgerecht gestaltet und speziell ausgebildete Betreuungskräfte eingestellt. Nicht erst seit im Jahr 2014 bei der Deutschen Alzheimer-Gesellschaft „Demenz im Krankenhaus“ ein Schwerpunktthema war, wurde öffentlich kritisiert, dass verwirrte Patienten auf „normalen“ Stationen verloren gingen. Seitens der Kliniken wird von „störendem“ Verhalten gesprochen, selbst wenn es sich nur um Symptome einer Erkrankung handelt.

Das Versorgungsnetz in der Alterspsychiatrie ist ein gänzlich anderes als das in der Allgemeinpsychiatrie oder Sucht. Komplementärer Partner ist die Altenhilfe, die inzwischen sehr gut und differenziert ausgebaut ist. Es wäre eine Herkulesaufgabe, wenn vor allem Ärzte und Sozialarbeiter einer gemischten psychiatrischen Station gleichzeitig das Suchthilfenetz, den gemeindepsychiatrischen Verbund mit dem Schwerpunkt bei den Psychoseerkrankten, das Kompetenznetz Depression und die komplette Altenhilfe in ihrer Region gut kennen und entsprechende Kooperationsstrukturen mit allen Einrichtungen entwickeln müssten. Angesichts der niedrigen Verweildauern in den deutschen Alterspsychiatrien und der steigenden Anzahl an Betroffenen (die Altersgruppe der über 65-Jährigen ist in den letzten 25 Jahren um rund 50\% gewachsen) ist eine aufsuchende Arbeit in den Pflegeheimen zwingend, um für Erstaufnahmen (beispielsweise in der alterspsychiatrischen Abteilung unserer Klinik über $80 \%$ ) leistungsfähig zu bleiben. Auch diese aufsuchende Arbeit ist nur durch eine enge Vernetzung spezialisierter Stationen mit der korrespondierenden Ambulanz möglich. Schließlich geht es um Mitarbeit in regionalen Gremien und Hilfenetzwerken. Auch die kann in der erforderlichen Differenziertheit und mit dem nötigen Engagement nicht in allen psychiatrischen Netzwerken erfolgen.

Das gleiche Argument gilt auch für den Stand des medizinischen und pflegerischen Wissens, die fachspezifische Weiterbildung und die spezielle Angehörigenarbeit, die sich z.B. im Suchtbereich und bei Demenzpatienten ganz erheblich unterscheidet. Eine profunde Beschäftigung mit Betreuungsrecht, Patientenverfügungen, ethischen und rechtlichen Fragen am Lebensende gelingt nur dann, wenn sie regelmäßig mit spezifischer Erfahrung supervidiert wird.

Architekturkonzepte stellen einen wichtigen Wirkfaktor im Milieu dar und müssen daher bei Sanierungen und Neubauten auch in psychiatrischen Kliniken beaufsichtigt werden. Es geht um Aspekte von Beleuchtung, irritierende Oberflächen (dunkler Boden wird als Grube empfunden, spiegelndes Glas täuscht ein Gegenüber vor), Übersichtlichkeit, Gartenanlagen etc. Es braucht intelligente, der Klientel angepasste Konzepte für die Türöffnung, Verwirrte haben grundsätzlich andere Bedürfnisse als orientierte und absprachefähige Patienten [2]. Auch der Tagesablauf wird den Bedürfnissen dieser Patientengruppe angepasst. Ein ausgiebiges Frühstück spielt eine tragende Rolle bei der Deckung des Energiebedarfs und hat damit z.B. Vorrang vor Aktivierungsangeboten zur gleichen Uhrzeit. Eine derartige „Ungleichbehandlung“ wäre anderen Patientengruppen kaum zu vermit- 
teln. Die Gruppenangebote selbst sind kurz und berücksichtigen damit die begrenzte Aufmerksamkeitsspanne. Suchtpatienten wären auch kaum mit einer Erinnerungsgruppe über ihre Schulzeit zufrieden oder damit, sich einen Wasserball zuzurollen, um überhaupt in Kontakt zu kommen. Die Unterschiede mit Spaziergängen, oft auch im Rollstuhl, setzen sich fort bis zum Abend, wenn die Konzentrationsfähigkeit nachlässt und Präsenzkräfte vis-à-vis Kontakt halten oder noch einmal zum Mitsingen auffordern.

Die Hoffnung, dass eine Mischung von Patienten aller Diagnosen und Altersgruppen nicht nur ein Spiegel der Gesellschaft ist, sondern alltägliche Kontaktangebote von diesen Patienten übernommen werden, trügt in mehrfacher Hinsicht. Zum einen sind im Alltaggespräch normal kommunikative Demenzpatienten die Ausnahme, denn diese werden inzwischen nahezu ausschließlich ambulant behandelt. Zum anderen sprechen Aufnahmeanfragen benachbarter gemischter Einheiten mit den Argumenten, dass die „gesünderen und jüngeren“ sich vom Verhalten der Demenzkranken gestört fühlen („Gebiss in der Suppe des Nachbarn“, nächtliches Rufen, Räumen, Urinieren in fremde Zimmer) gegen diese gesellschaftliche Wunschvorstellung. Schließlich sind auch in der Gesellschaft altersgleiche Gruppen eher die Regel als die Mischung von Generationen, man denke an Schule, Ausbildung, Sport, Arbeit, selbst in Vereinen sind selten zwei Generationen vertreten.

Ein demenzspezifisches Behandlungsmilieu ist wirksam. Es ist wichtig, die Auf- merksamkeit auf die durch die nachlassende Sinneskraft entstehenden Einschränkungen zu richten, nämlich die Versorgung mit Hörgeräten und Sehhilfen zu gewährleisten. Zum Wohlbefinden tragen auch ein gezieltes Gehtraining und nächtliche Angebote wie ein Nachtcafé bei. Verwirrte Patienten im psychiatrischen und Allgemeinkrankenhaus stellen einen nicht unbeträchtlichen, tendenziell wachsenden Anteil an Patienten dar, deren Bedürfnisse immer wieder zu wenig beachtet werden. Alle Untersuchungen zur Milderung von verwirrten und deliranten Syndromen haben gezeigt, dass störungsspezifische Angebote und Behandlungsformen wirksam sind, oft wirksamer als pharmakologische Interventionen. Dies ist in der gebotenen Qualität und Intensität nur in spezialisierten Behandlungseinheiten möglich und wurde bestätigt in speziellen Behandlungseinheiten an internistischen und geriatrischen Kliniken [3-5].

Daher haben in den letzten 25 Jahren alle Fachkrankenhäuser und viele große psychiatrische Abteilungen in Allgemeinkrankenhäusern, die genügend Betten vorhalten, Behandlungseinheiten für Delir- und Demenzpatienten eingerichtet. Die Weiterentwicklung wird sein (was an einigen Orten schon Realität ist), derartige Einheiten kooperativ mit Innerer Medizin, Geriatrie oder Neurologie zu betreiben, um die Expertise aus den genannten Fachgebieten, die sich ergänzen, den Patienten zur Verfügung zu stellen.

\section{Literatur}

1 Kortus R, Kipp J. Integration oder Separation Demenzkranker in der Psychiatrie - sind „Demenzstationen sinnvoll? In: Wächtler C, Hirsch RD, Kortus R, Stoppe G, Hrsg. Demenz. Die Herausforderung. Tagungsband des DGGPP-Kongresses 1995. Singen: Verlag Egbert Ramin; 1996: 170-194

2 Renteln-Kruse W, Neumann L, Klugmann B et al. Kognitiv beeinträchtigte geriatrische $\mathrm{Pa}$ tienten - Patientenmerkmale und Behandlungsergebnisse auf einer spezialisierten Station. Dtsch Arztebl Int 2015; 112: 103 122

3 Hofmann W, Rösler A, Vogel $W$ et al. Special care units for acutely ill patients with cognitive impairment in Germany. Position paper. Z Gerontol Geriatr 2014; 47: 136-140

4 Flaherty JH, Little MO. Matching the environment to patients with delirium: lessons learned from the delirium room, a restraintfree environment for older hospitalized adults with delirium. J Am Geriatr Soc 2011; 59 (Suppl. 02): S295-300

5 Hshieh TT, Yue J, Oh E et al. Effectiveness of multicomponent nonpharmacological delirium interventions: A Meta-analysis. JAMA Intern Med 2015; (published online)

Korrespondenzadresse

Dr. Jochen Tenter

Leiter der Abteilung für Alterspsychiatrie und Psychotherapie, Zentrum für Psychiatrie Südwürttemberg, Ravensburg-Weissenau Weingartshofer Straße 2

88214 Ravensburg

jochen.tenter@zfp-zentrum.de

Bibliografie

DOI http://dx.doi.org/

10.1055/s-0034-1387617

Psychiat Prax 2015; 42: 179-180

(c) Georg Thieme Verlag KG

Stuttgart · New York

ISSN 0303-4259 